\title{
Competitividad empresarial de las pequeñas y medianas empresas manufactureras de Baja California
}

\author{
Business competitiveness in the small and \\ medium-sized medium enterprises of the \\ manufacturing sector in Baja California
}

Recibido el 11 de enero de 2016. Aceptado el 1 de noviembre de 2016.

Autor para correspondencia: Manuel Alejandro Ibarra Cisneros, correo electrónico: manuel_ibarra@uabc.edu.mx

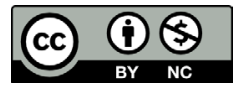

Todos los contenidos de Estudios Fronterizos se publican bajo la licencia Creative Commons Atribución no comercial 2.5 México, y pueden ser usados gratuitamente para fines no comerciales, dando el crédito a los autores y a la revista Estudios Fronterizos.
Manuel Alejandro Ibarra Cisneros ${ }^{\mathrm{a}^{*}}(\mathrm{DD})($ http://orcid.org/0000-0002-0731-7225) Lourdes Alicia González Torres ${ }^{\mathrm{a}}{ }^{(\mathbb{D}}$ (http://orcid.org/0000-0003-4725-0544) María del Rosario Demuner Flores ${ }^{b}$ (iD (http://orcid.org/0000-0002-4542-9113)

\footnotetext{
${ }^{a}$ Universidad Autónoma de Baja California, Facultad de Ciencias Administrativas, Baja California, México, correo electrónico: manuel_ibarra@uabc.edu.mx, alicia.gonzalez@uabc.edu.mx

${ }^{\mathrm{b}}$ Universidad Autónoma del Estado de México, Facultad de Contaduría y Administración, México, correo electrónico: demuner7@yahoo.com
}

\section{Resumen}

El objetivo de esta investigación es determinar el nivel de competitividad empresarial de las pymes manufactureras de Baja California e identificar las áreas que dentro de ellas, influyen en dicha competitividad. La metodología es de carácter descriptivo, correlacional y de corte transversal; se desarrolló el modelo de competitividad sistémica a escala micro y se aplicó un instrumento de medición de 64 preguntas a 195 empresas del estado; además, se hizo uso de regresiones lineales múltiples de tipo tradicional para la comprobación de hipótesis. Entre los hallazgos tenemos que las pymes en el estado presentan un nivel de competitividad medio-bajo, sin mostrar relación entre el tamaño de las empresas y su competitividad, mientras que el área de producción-operaciones resultó ser más significativa para su nivel de competitividad. Si bien el instrumento de medición que se aplicó no tiene proporcionalidad a nivel de municipios y subsector, sí nos permite acercarnos al funcionamiento interno de las pymes.

Palabras clave: competitividad sistémica, pequeñas y medianas empresas, manufactura.

\section{Abstract}

The objective of this research is to determine the competitiveness level of the manufacturing small and medium-sized enterprises (SMEs) of Baja California and to identify which areas within them affect this competitiveness. The meth-

CÓMO CITAR: Ibarra, M. A., González, L. A. y Demuner, M. del R. (2017). Competitividad empresarial de las pequeñas y medianas empresas manufactureras de Baja California [Business competitiveness in small and medium-sized enterprises of manufacturing sector in Baja California]. Estudios Fronterizos, 18(35), 107-130, doi:10.21670/ref.2017.35.a06 
odology used is descriptive, correlational, and cross-sectional; the systemic competitiveness model is developed at the micro level, and a measurement instrument with 64 questions is used on 195 companies in the state; in addition, traditional multiple linear regressions are performed to test the hypotheses. One of the findings is that the SMEs in the state have a medium-low competitiveness level and do not show any relationship between the size of the companies and their competitiveness; however, the production-operations area prove to be more important for their competitiveness level. Although the measurement instrument that is used has no proportionality at the municipality and subsector level, it does allow us to approach the internal operation of the sMEs.

Keywords: systemic competitiveness, small and medium-sized enterprises, manufacturing.

\section{Introducción}

El estudio de la competitividad se ha convertido en una línea de investigación cada vez más trascendente no solo entre los académicos sino también entre las organizaciones. Su importancia radica en que puede ser analizada desde diversas perspectivas y todas ellas contribuyen a revelar una parte de la competitividad que se genera en una organización. Existen numerosas definiciones sobre la competitividad desde el nivel empresa, hasta el nivel de países. Una de las más importantes y complejas es la competitividad sistémica, la cual, analiza desde los niveles macroeconómicos, políticas de desarrollo económico, factores culturales y sociales, desde el nivel empresa hasta escala industria.

Por otra parte, las pequeñas y medianas empresas (pymes) manufactureras en el país y particularmente en la región de Baja California, México - la cual está conformada por cinco municipios: Ensenada, Mexicali, Playas de Rosarito, Tecate y Tijuana—, son la base del tejido industrial del estado, ya que son generadoras de una parte significativa del empleo formal así como de su contribución al crecimiento económico. Baja California, al ser un estado fronterizo se caracteriza por su vocación industrial destacando las industrias aeroespacial, electrónica, metal-mecánica, de insumos médico y automotriz. Por esta razón, la industria manufacturera contribuye con $54 \%$ del producto interno bruto (РIB) del sector industrial, destacando el de maquinaria y equipo. De ahí que la existencia de una industria manufacturera competitiva permitirá que la contribución de esta al PIB estatal se siga fortaleciendo y genere mayor cantidad de empleo y riqueza tanto al estado como al país. Se dice que el sector manufacturero en el estado es competitivo, pero surge la interrogante ¿qué tan competitivo es? Por otra parte, tampoco se conoce qué áreas dentro de una empresa permiten coadyuvar a la competitividad empresarial de las mismas.

El primer objetivo del presente trabajo consiste en determinar el nivel de competitividad a nivel empresarial o micro de las pymes manufactureras de Baja California; mientras que el segundo consiste en determinar qué áreas dentro de las empresas influyen más sobre tal nivel de competitividad, para lo cual, se plantean hipótesis para cada área y se plantea como hipótesis adicional la existencia de una relación positiva entre el tamaño de la empresa y el nivel de competitividad de las mismas. Para alcanzar dichos objetivos y responder a tales interrogantes, se diseñó un instrumento de medición utilizando las ocho dimensiones vertidas en el modelo de competitividad sistémica del Banco Interamericano de Desarrollo (BID) a nivel de empresa, el cual fue aplicado a empresas de todos los subsectores manufactureros del estado. El análisis de resultados se divide en dos partes, la primera de manera descriptiva, permite establecer el nivel de competitividad de las 
pymes manufactureras; y la segunda de manera inferencial, mediante la utilización de regresiones lineales múltiples de tipo tradicional para encontrar relaciones positivas entre las ocho dimensiones o áreas dentro de una empresa que contribuyen a la competitividad empresarial.

La contribución de esta investigación al estado del arte radica en que al tener mayor conocimiento del funcionamiento interno de las pymes del sector, estas podrán realizar acciones para disminuir determinadas falencias que están bajo su control y permitir elevar sus niveles de eficiencia, productividad y por tanto, competitividad. Reconociendo que la competitividad es sistémica, se deben analizar los diferentes ámbitos en los que se desenvuelve una empresa -micro, macro, meta y meso—; sin embargo, este estudio se centra en el nivel micro, por ser el único donde el empresario tiene injerencia directa y puede controlar.

El presente trabajo está dividido en cuatro secciones. La primera abarca el marco contextual de las pymes manufactureras en Baja California, pasando por una exploración de la competitividad, concepciones e implicaciones desde el ámbito internacional al ámbito empresarial; desde luego analizando también lo referente a la competitividad sistémica y diversos estudios en esta materia realizados en el país. La segunda, corresponde en explicar la metodología utilizada que da cumplimiento al objetivo de esta investigación. En la tercera se hace un análisis de datos y discusión de resultados que permiten dar respuesta a los objetivos e hipótesis planteados. Finalmente, se abordan una serie de conclusiones y líneas de investigación que pueden derivar de la presente investigación.

\section{El contexto de las pymes del sector manufacturero en Baja California}

Las pequeñas y medianas empresas son las organizaciones dominantes en todos los países del continente, representan más de 95\% de los negocios existentes en el mundo y cuentan con algunas ventajas competitivas con respecto a las grandes empresas, debido a su menor tamaño y facilidad de adaptarse a los cambios en la economía y patrones de la demanda (González, 2013). Además de ser dominantes, son parte fundamental en la creación de empleo en todos los países, ya que gran parte del mismo es generado gracias a ellas, así como su contribución al crecimiento económico (Vázquez y Arredondo, 2014).

Según el Instituto Nacional de Estadística y Geografía (Inegi) (2009), las empresas en México se clasifican de acuerdo al número de trabajadores que se empleen en ellas; en el caso de las pequeñas empresas del sector industrial el número de empleados oscila entre 11 y 50, las medianas se conforma de 51 a 250 trabajadores. De acuerdo con ProMéxico (2013, párr. 1), las pymes constituyen la columna vertebral de la economía nacional por los acuerdos comerciales que ha tenido México en los últimos años y también por su alto impacto en la generación de empleos y en la producción nacional. Según Inegi (2014a), en México existen aproximadamente 4015000 unidades empresariales, de las cuales 99.8\% son micro, pequeñas y medianas empresas - mipymes- que generan $52 \%$ del PIв y 72\% del empleo (ProMéxico, 2013, párr. 1), consecuentemente las pymes se han convertido en una importante fuente de trabajo en el país.

Adicionalmente, la pyme manufacturera es responsable de $50 \%$ de las exportaciones de México en este segmento. De los 2500 millones de dólares que el país exportó en productos de las pymes durante 2011, la mitad correspondió a empresas que no solo fabrican manufacturas para proveer al mercado doméstico, sino también para el exterior (Rosagel, 2012). En el país, existen alrededor de 250000 pymes, entre ellas destacan las 
manufactureras. Dentro del total de pymes contabilizadas en el país, el sector servicios es el más representativo con 157153 unidades económicas; dentro del sector industrial se identificaron un total de 29835 empresas, representando cerca de $12 \%$ del total de unidades económicas existentes en el país dentro de este colectivo. Esta misma proporción se mantiene con respecto a las unidades económicas existentes en Baja California, donde existe un total de 1004 pymes manufactureras de las 8709 en todo el estado (Inegi, 2014a).

Al realizar un análisis desagregado por número de pymes en Baja California, de acuerdo con Inegi, Tijuana posee $52.49 \%$ de las pymes manufactureras con un total de 527. Seguido de Mexicali (226) y Ensenada (149), de manera marginal se encuentran el resto del estado con apenas $10 \%$. Por subsectores manufactureros, en el estado destacan las de productos alimenticios, bebidas y tabaco (180), maquinaria y equipo (174) e industria metálica básica y productos metálicos (136). Esta tendencia se presenta en los municipios de Tijuana, Mexicali y Tecate; por su parte, en Ensenada predomina la industria textil seguido de la alimentaria.

Datos del Inegi (2014b) indican que en Baja California, el sector industrial representó 35.18\% del PIB estatal en 2013, con un total de \$134 931000 pesos, de los cuales corresponden $\$ 73726000$ a la industria manufacturera (54.6\%) del PIB industrial. Dentro de esta industria el subsector que más aporta es el de maquinaria y equipo con $41 \%$, seguido de la industria alimentaria, de las bebidas y el tabaco y otras industrias. Estos datos muestran la especialización industrial del estado al poseer fuertes industrias como la aeroespacial, productos médicos y electrónicos principalmente, donde inclusive se destaca a escala internacional (Investinbaja, s.f.; Oxford Bussines Group, 2014).

\section{La competitividad: De la escala internacional a la micro o empresarial}

Actualmente el concepto de competitividad ha adquirido una gran presencia en la literatura tanto internacional como a escala local al momento de analizar el progreso económico tanto de países como de empresas. Comparativos internacionales permiten indagar que naciones - por medio de sus empresas - han brindado mejoras a sus ciudadanos a través del incremento en el nivel de vida. Por tanto, se reconoce que el nivel de competitividad internacional de un país se relaciona estrechamente con empresas altamente productivas que aprovechan las ventajas competitivas que genera el país por medio de sus instituciones, políticas, infraestructura y cultura.

Saavedra y Milla (2012, párr. 16) comentan que:

El término competitividad no posee una definición específica. Existe una falta de consenso para definir[la] conceptualmente [...], debido a la amplitud de su significado, que puede abarcar desde el nivel de la empresa, sector, nación y ámbito supranacional; así como, por la naturaleza cualitativa y cuantitativa de sus factores carece de límites precisos en el nivel de análisis y en las diversas metodologías de medición.

No existe una definición única para este concepto, es por ello que se despliegan una serie de definiciones para ampliar el sentido y significado de la misma desde diversos enfoques. Para el World Economic Forum (2010, p. 4), el concepto de competitividad es "el conjunto de instituciones, políticas y factores que determinan el nivel de productividad 
de un país". El incremento en dicha productividad permitirá que el nivel de ingresos de un país aumente, logrando a su vez, prosperidad para los ciudadanos mediante un incremento en los niveles de calidad de vida.

Otro concepto similar al del Foro Económico Mundial es el de Dussel (2001, p. 11), que define la competitividad como "el proceso de integración dinámica de países y productos a mercados internacionales [...], dependiendo tanto de las condiciones de oferta como de las de demanda"; mientras que Porter (1991), sostiene que la competitividad de una nación se debe a que sus empresas son altamente productivas gracias al uso eficiente de sus recursos humanos, naturales y de capital. Para lo cual diseñó el modelo — diamante de competitividad- que permite visualizar las ventajas competitivas de un país en relación con otros; aunque también se puede llevar a cabo a escala industria o empresa, por medio del análisis de las condiciones de los factores, de la demanda, estrategia, estructura y rivalidad empresarial, así como las industrias relacionadas y de apoyo. La conjugación de estos factores da como resultado el nivel de competitividad que se presenta en una economía, así es posible determinar qué elementos dentro de cada factor, son más relevantes al momento de impulsar la productividad de las empresas que a largo plazo las llevan a un mayor nivel de competitividad. Por su parte, la Organización para la Cooperación y el Desarrollo Económico (ocDE, 1992), ha desarrollado diversos estudios para identificar los principales enfoques de la competitividad logrando compendiar el concepto de "competitividad estructural", en el cual se identifican tres factores:

[a] La innovación como elemento constitutivo central del desarrollo económico; [b] la capacidad de innovación de una organización industrial, situada fuera de las teorías tayloristas, de desarrollar capacidades propias de aprendizaje, y $[c]$ el papel de las redes de colaboración orientadas a la innovación y apoyadas por diversas instituciones, para fomentar las capacidades de innovación (Hernández, 2001, p. 15).

Asimismo, Labarca (2007, p. 161) traduce la competitividad en la "posibilidad que tienen sus ciudadanos para alcanzar un nivel de vida elevado y creciente; [el cual, está] determinado por la productividad con la que se utilizan los recursos nacionales, el producto por unidad de trabajo o el capital utilizado". Además, la competitividad se logra "alcanzando una mayor productividad en los negocios existentes o incursionando exitosamente en negocios de mayor productividad" (Labarca, 2007, p. 161). Esta definición va más acorde a lo planteado por el World Economic Forum (2010) y a las ideas de Porter (1991), donde las variables productividad y calidad de vida son fundamentales como parte de la ecuación.

Dentro de las diversas definiciones de competitividad, se identifica la denominada competitividad empresarial. De igual manera que en el punto anterior, existe la imposibilidad de establecer una sola concepción; sin embargo, las definiciones vertidas por diversos autores tienden a desagregar el tema de la competitividad internacional y focalizarlo al plano nacional-local al interpretar que la competitividad empresarial es una parte integrante de la competitividad de los países y por tanto, no son excluyentes. Al respecto, Abdel y Romo (2004, p. 9) enfatizan que:

La competitividad empresarial se deriva de la ventaja competitiva que tiene una empresa a través de sus métodos de producción y de organización (reflejados en precio y en calidad del producto final) en relación con los de sus rivales en un mercado específico. 
A lo que otros autores agregan:

La factibilidad de que una empresa alcance y mantenga sus niveles de competitividad se concentra en las competencias distintivas o ventajas competitivas que desarrolle internamente y en los condicionamientos externos que le brindan tanto la industria o sector al que pertenece, como la región-país en la que se encuentra ubicada (Cabrera-Martínez, López-López y Ramírez, 2011, p. 25).

Se concuerda con el punto de vista de Flores y González (2009, p. 88):

El éxito empresarial descansa en la capacidad organizativa de anticiparse y reaccionar a las exigencias de los mercados, [por lo que] un factor clave de dicho éxito es la flexibilidad, [entendida como] la capacidad del empresario de adaptarse de manera rápida a los cambios que demanda el mercado.

Por último, Suñol (2006, p. 184) menciona que es imprescindible:

La creación de competitividad sistémica, [la cual] se basa en tres pilares: [a] el desarrollo de sistemas de innovación que aceleren la acumulación de capacidad tecnológica, $[b]$ el apoyo a la diversificación y la creación de encadenamientos productivos, y $[c]$ la provisión de servicios de infraestructura de calidad.

Asimismo, establece que en el entorno de la empresa, se conjugan gran cantidad de factores que inciden directa o indirectamente en el nivel de competitividad de la empresa, como son el nivel educativo, la infraestructura, la sofisticación del mercado de productos, la cultura empresarial, la eficiencia de las instituciones y el medio ambiente, por mencionar algunos.

Para la ocDe (citado en Cabrera-Martínez et al., 2011, pp. 23-24) los indicadores de la competitividad empresarial se basan en los siguientes aspectos:

a) La exitosa administración de los flujos de producción y de inventarios de materia prima y componentes.

b) La integración exitosa de planeación de mercado, actividades de investigación y desarrollo - I+D-, diseño, ingeniería y manufactura; el proceso de innovación es uno de los principales pilares para sustentar competitividad y el desarrollo económico, las patentes y otras formas de protección industrial e intelectual, juegan un papel decisivo para el crecimiento.

c) La capacidad de combinar $\mathrm{I}+\mathrm{D}$ interna con $\mathrm{I}+\mathrm{D}$ realizada en universidades, centros de investigación y otras empresas.

d) La capacidad de incorporar cambios en la demanda y la evolución de los mercados así como la generación de un ambiente propicio para los negocios es un factor importante para la prosperidad económica, la competitividad y el crecimiento.

e) Fomento de los programas productivos mediante esquemas que facilitan una mayor integración y asociaciones entre las empresas. Establecimiento de esquemas en los cuales se permita el desarrollo entre proveedores y distribuidores dentro de la cadena de valor.

Berumen (2006) agrupa los determinantes de la competitividad de la empresa en dos tipos. Los primeros son los relacionados con los precios y los costos; mientras que los segundos están relacionados con la calidad de los productos, la incorporación de mejoras 
tecnológicas en los procesos, "gestión eficiente de los flujos de producción, capacidad para desarrollar y mantener relaciones con otras empresas" (Cabrera-Martínez et al., 2011, p. 24), y la vital capacidad de generar procesos de investigación, desarrollo e innovación $(\mathrm{I}+\mathrm{D}+\mathrm{I})$, entre otros.

\section{Competitividad sistémica}

De acuerdo con Morales y Castellanos (2007, p. 33), últimamente:

En la región latinoamericana ha crecido el interés por adoptar esquemas integrales que permitan entender y propiciar un desarrollo dinámico de los aspectos que afectan la competitividad. Es así que países como Brasil y México han adaptado el modelo de competitividad sistémica de acuerdo con un enfoque particular al tener en cuenta las especificidades de cada sector a través de la ponderación diferenciada y dando mayor importancia a los aspectos que se consideran estratégicos.

El estudio realizado por Esser, Hillebrand, Messner y Meyer-Stamer (1996, p. 40) analiza el concepto de competitividad sistémica, estableciendo un marco de referencia tanto para los países industrializados como para los que están en vías de desarrollo y tiene como premisa "la integración social, exigiendo reformas económicas y un proyecto de transformación de la sociedad" (Messner citado en Saavedra y Milla, 2012, p. 21). Se distingue por incluir "cuatro niveles analíticos distintos: meta, macro, meso y micro" (Messner, 1996, párr. 39). En el nivel meta se estudian los factores como la capacidad de una sociedad en términos de integración y estrategia, así como consenso con "orientación al mercado y al mercado mundial" (Esser et al., 1996, p. 42). El nivel meso examina la formación de un entorno con la capacidad de fomentar, complementar y multiplicar los esfuerzos al nivel de la empresa, tales como la fusión de los elementos que forman parte de la economía industrial, de la teoría de la innovación y de la sociología industrial. El nivel macro hace referencia a la estabilización del contexto macroeconómico apoyándose en las reformas de las políticas fiscal y presupuestaria, así como de la monetaria y cambiaria (Messner, 1996, párr. 39).

Labarca (2007, p. 161) menciona que en el plano nacional:

La competitividad se medirá por el nivel de vida alcanzado y su crecimiento, el nivel y el crecimiento de la productividad agregada y la capacidad de las empresas nacionales para aumentar su penetración en los mercados mundiales a través de exportaciones o inversiones extranjeras directas.

En este sentido, Suñol (2006) argumenta que el éxito de las empresas competitivas a escala internacional viene precedido de una prolongada estabilidad macroeconómica en el país de origen, que a su vez, permite crear un ambiente macroeconómico competitivo gracias a "la disponibilidad de recursos físicos, naturales, institucionales y humanos" (Suñol, 2006, p. 196), los cuales al ser bien administrados a largo plazo, son catalizadores bajo los cuales las empresas pueden competir globalmente.

En el nivel microeconómico se ha generado un mejoramiento continuo de las empresas y las cadenas de producción, con ello se han producido externalidades positivas $[\ldots]$ y se ha generado un entorno en el que las empresas desarrollan cuatro factores necesarios para alcanzar la competitividad: eficiencia en costos, 
calidad, diversidad de productos y capacidad de respuesta; para lograrlo se han realizado cambios en [...] organización de la producción, desarrollo de producto y organización de la cadena de valor (Gracia, 2006, p. 41).

Como apuntan Cabrera-Martínez et al. (2011, p. 17):

La competitividad no surge al modificarse el contexto macro ni se crea recurriendo exclusivamente al espíritu de empresa a nivel micro. La competitividad es, [...], el producto de un patrón de interacción compleja y dinámica entre el Estado, las empresas, las instituciones intermedias y la capacidad organizativa de una sociedad.

[En] términos de enfoque sistémico la competitividad empresarial depende de la interacción entre los elementos del nivel micro con aquellos del nivel meso, macro y meta. Asimismo, se requiere que las empresas alcancen un alto nivel de productividad, calidad, flexibilidad y agilidad, que les permita sostener una ventaja competitiva estratégica y generar redes empresariales para acelerar los procesos de aprendizaje colectivo (Saavedra y Milla, 2012, p. 28).

Adicionalmente, el éxito de una organización radica en parte de las estrategias y políticas en favor de su competitividad, las cuales deben ser de uso frecuente; además es indispensable que se incorporen mejoras en los procesos e innovaciones para mantenerse por encima de la competencia en el sector al que pertenece la organización, todo este tipo de acciones coadyuvarán en el nivel de competitividad de la empresa (Aragón y Rubio, 2005; Berumen, 2006; Estrada, García y Sánchez, 2009).

En el nivel de competitividad a nivel micro o empresarial se pueden distinguir ocho dimensiones (ver Tabla 1), las cuales de manera combinada determinan qué tan competitiva es una empresa así como el grado de éxito que esta presenta respecto al resto del mercado. Dichas dimensiones son las siguientes:

- Planeación estratégica: se refiere a si las organizaciones tienen objetivos y metas a plazo, políticas para su cumplimiento y seguimiento. Se considera si han realizado estudios sobre el entorno, amenazas y planes de contingencia. En teoría esta dimensión debería aplicarse a cabalidad en la totalidad de las empresas ya que en ella se sustenta el ser y hacer de las mismas; sin embargo, la evidencia muestra que en ocasiones esta no es considerada una prioridad que permita dar mayor competitividad a una empresa (Aragón, Rubio, Serna y Chablé, 2010; Castellanos, Gálvez, Montoya, Lagos y Montoya, 2006; Du y Banwo, 2015; Estrada et al., 2009; Rosto, 2010).

- Producción y operaciones: su importancia sobre la competitividad de la empresa radica en la complejidad de los procesos de producción, uso de herramientas modernas de producción, certificaciones, flexibilidad en procesos productivos, planeación de materias primas e insumos, desarrollo de nuevos productos, manejo de inventarios, entre otros factores. Estos permiten que las empresas puedan reaccionar en el corto plazo ante los cambios en los patrones de la demanda y factores externos a la organización; por tanto, a mayor flexibilidad y modernización de los procesos y operaciones, mayor será el nivel de competitividad de la empresa (Du y Banwo, 2015; Medina y Naranjo, 2014; Saavedra y Tapia, 2011; Zevallos, 2003).

- Aseguramiento de la calidad: en esta dimensión se determina el nivel de implementación de normas de calidad, grupos de trabajo al respecto, 
certificaciones, programas para enfrentar contingencias. La evidencia indica que aquellas empresas donde los procesos de calidad y la calidad de los productos son certificados y elevados, estas presentan mayores niveles de competitividad (Aragón et al., 2010; Flores y González, 2009).

- Comercialización: consiste en el análisis de las políticas de venta, vías de distribución, formas de pago, relación con clientes y proveedores, definición del mercado meta, estudios de mercado, uso de estrategias de mercadeo, satisfacción del cliente. Esta es una de las dimensiones más relevantes ya que la interacción con clientes y proveedores es esencial tanto para producir de manera eficiente como para distribuir y vender los productos entre los clientes de forma exitosa (Aragón y Rubio, 2005; Flores y González, 2009; Martínez, Palos, León y Ramos, 2013; Zevallos, 2003).

- Contabilidad y finanzas: se estudia si las empresas tienen definida su estructura de costos, margen de ganancias, planeación y administración financiera, estrategias fiscales, pago de impuestos, etc. Gran parte del éxito o fracaso de las organizaciones se relaciona con el acceso al financiamiento y las estrategias para hacer uso adecuado e inteligente de los recursos económicos de las organizaciones (Aragón y Rubio, 2005; Saavedra y Tapia, 2011; Zevallos, 2003).

- Recursos humanos: parte de la competitividad de una organización se relaciona con el adecuado uso del recurso humano, es esencial contar con un proceso riguroso de selección y contratación, programas de capacitación y adiestramiento, análisis de las causas de la rotación laboral, clima laboral y los programas para remediarlos, sistemas de compensaciones, cumplimiento de aspectos de seguridad e higiene industrial (Aragón et al., 2010; Aragón y Rubio, 2005; Flores y González, 2009; Martínez et al., 2013).

- Gestión ambiental: las nuevas exigencias en esta materia son de mayor relevancia para los consumidores, las empresas deben ser competitivas y a la vez responsables con el medio ambiente. Una empresa competitiva analiza el uso de normas ambientales, crea programas en la materia, tiene políticas de manejo de desechos, políticas de reciclaje, entre otras medidas (Castellanos et al., 2006).

- Sistemas de información: existe una amplia evidencia de la importancia de la tecnología como mecanismo de competitividad empresarial. Las organizaciones que son conscientes y adoptan las tecnologías de información y comunicación, cuentan con personal especializado, tienen un cierto grado de sistematización y generan planes de contingencia son más proclives a ser más competitivas a escala no solo nacional sino internacional (Aragón et al, 2010; Aragón y Rubio, 2005; Cuevas-Vargas, Aguilera, González y Servín, 2015; Estrada et al., 2009; Flores y González, 2009; Ibarra, González y Cervantes, 2014; Zevallos, 2003).

De acuerdo con la literatura referida anteriormente, es de esperar que cuanto mejor sea la planeación estratégica en las empresas, así como que estas cuenten con un adecuado sistema de gestión del medio ambiente, adopten sistemas de información y manejen de manera eficiente sus recursos humanos, tendrán mayor capacidad de tomar medidas que incrementen su competitividad.

Las ocho dimensiones descritas anteriormente abarcan múltiples actividades que se realizan de manera cotidiana y sistemática en las empresas, por lo cual se considera que son las adecuadas para llevar a cabo el análisis de competitividad empresarial en las pymes manufactureras de Baja California. Bajo esta estructura de análisis, todas las áreas de las empresas son tomadas en consideración de manera igualitaria, lo que permite generar resultados que describan el comportamiento de cada área y su contribución a 
la competitividad global de la empresa. Además, la amplia literatura generada hasta el momento, respalda la necesidad de integrar todos los aspectos de una empresa para evitar generar sesgos entre áreas y actividades que afecten los resultados reales de los análisis de competitividad que se lleven a cabo.

Tabla 1: Dimensiones de la competitividad empresarial

\begin{tabular}{|c|c|}
\hline Planeación estratégica & $\begin{array}{l}\text { Objetivos } \\
\text { Metas } \\
\text { Políticas } \\
\text { Análisis del entorno } \\
\text { Planes de contingencia }\end{array}$ \\
\hline Producción y operaciones & $\begin{array}{l}\text { Procesos de producción } \\
\text { Certificaciones } \\
\text { Flexibilidad productiva } \\
\text { Desarrollo de nuevos productos y procesos } \\
\text { Planeación de materiales, insumos, etc. }\end{array}$ \\
\hline Aseguramiento de la calidad & $\begin{array}{l}\text { Normatividad } \\
\text { Grupos de trabajo y retroalimentación } \\
\text { Procesos certificados }\end{array}$ \\
\hline Comercialización & $\begin{array}{l}\text { Políticas de venta } \\
\text { Distribución } \\
\text { Relaciones clientes-proveedores } \\
\text { Satisfacción de clientes } \\
\text { Investigación de mercados }\end{array}$ \\
\hline Contabilidad y finanzas & $\begin{array}{l}\text { Estructura de costos } \\
\text { Administración financiera } \\
\text { Estrategias fiscales } \\
\text { Pago de impuestos } \\
\text { Inventarios }\end{array}$ \\
\hline Recursos humanos & $\begin{array}{l}\text { Procesos de selección y reclutamiento } \\
\text { Capacitación y adiestramiento } \\
\text { Rotación y clima laboral } \\
\text { Seguridad e higiene } \\
\text { Compensaciones }\end{array}$ \\
\hline Gestión ambiental & $\begin{array}{l}\text { Programa de manejo de desechos } \\
\text { Políticas de reciclaje } \\
\text { Normatividad }\end{array}$ \\
\hline Sistemas de información & $\begin{array}{l}\text { Tecnologías de la información } \\
\text { Sistematización } \\
\text { Planes de contingencia }\end{array}$ \\
\hline
\end{tabular}

Fuente: Elaboración propia con información de varios autores. 


\section{Evidencia empírica para el caso de México}

En México, diversos estudios empíricos se han llevado a cabo en la última década para conocer el nivel de competitividad de las empresas, particularmente mipymes, las cuales como se ha comentado anteriormente, son las principales generadoras de empleo en el país; por consiguiente, este apartado tiene por objetivo identificar a través de una revisión de estudios realizados qué áreas o dimensiones dentro de las empresas contribuyen en mayor medida a la competitividad de las mismas. De esta manera, los resultados encontrados permiten identificar puntos de coincidencia con el estudio efectuado en las pymes manufactureras de Baja California y así arribar a explicaciones para el análisis en cuestión. Desde el enfoque sistémico a nivel micro, se puede destacar el estudio de Aragón et al. (2010) donde se establece que los principales elementos de competitividad de las mipymes de Tabasco son: la aplicación de recursos tecnológicos, la innovación, la calidad de los productos y los recursos humanos. Asimismo, se encontró que aquellas empresas con un perfil exportador tienden a un mayor nivel de competitividad. En este sentido, Aragón y Rubio (2005) confirman los elementos de capacidad financiera, tecnologías, innovación, mercadotecnia y recursos humanos como los factores explicativos del éxito competitivo de las pymes del estado de Veracruz.

Flores y González (2009) al analizar las pymes de Morelia determinaron que la competitividad se asocia principalmente con los factores de relaciones con el exterior y la calidad de los productos; mientras que los factores recursos humanos y tecnología tienen menor grado de influencia. Martínez, Palos, León y Ramos (2013), señalan que los factores más importantes para la competitividad de las pymes en San Luis Potosí son la mercadotecnia, capital humano, política de costos y relación con proveedores. De igual manera se suman los estudios de Vázquez, Guerrero y Núñez (2014), para los estados de Aguascalientes, Colima, Jalisco y Querétaro, destacando el factor de tecnologías de la información. Mientras que los factores de planeación estratégica, innovación en productos, procesos y gestión, así como la tecnología presentaron una correlación positiva con respecto al éxito competitivo en las pymes del estado de Hidalgo (Estrada et al., 2009).

Por otra parte, la Red pymes-Cumex (2010) concluye que dentro de las pequeñas empresas de los estados de Estado de México, Hidalgo, Puebla y Sonora los elementos con mayor problemática son la planeación administrativa, recursos humanos y uso de las tecnologías de la información; sin embargo, el aspecto financiero fue el mejor catalogado. De acuerdo con Rosto (2010), la falta de una planeación estratégica de mercado impide la competitividad de las empresas en México, así como el modelo de administración de los dueños o gerentes. En el caso de los municipios de Torreón, Frontera y Monclova en el estado de Coahuila, el aspecto operacional y de mercadeo en las empresas es el más problemático para la sobrevivencia de las mismas, mientras que en la fase de consolidación el aspecto financiero es el que toma mayor relevancia (Molina, Armenteros, Medina, Barquero, Espinoza, 2011).

Adicionalmente, la investigación de Saavedra y Milla (2012) sobre la competitividad a nivel micro de las mipymes del estado de Querétaro, arrojó que en las ocho dimensiones se presentan bajos niveles de competitividad en la empresas del sector industrial; sin embargo, logran destacar los subsectores de industria química, de plástico y hule, así como el de industria metálica básica; en contraste, el de industria alimentaria fue el menos competitivo. 
De esta manera, se puede apreciar cómo los aspectos tecnológicos, la innovación, la calidad de los productos, el capital humano y la comercialización juegan un papel trascendental en el nivel de competitividad y éxito en las empresas de este país.

Analizado lo anterior, y con el propósito de determinar qué dimensiones de las ya mencionadas influyen sobre la competitividad empresarial de las pymes manufactureras, se plantean las siguientes hipótesis:

H1: la planeación estratégica incide sobre la competitividad de las empresas.

H2: las actividades de producción y operación influyen en la competitividad de las pymes.

H3: el aseguramiento de la calidad en las pymes permite que estas sean competitivas.

H4: las actividades de comercialización afectan directamente a la competitividad de las empresas.

H5: las actividades de contabilidad y finanzas inciden en la competitividad de las empresas.

H6: la gestión de recursos humanos se asocia al nivel de competitividad empresarial.

H7: la dimensión gestión ambiental influye en la competitividad de las pymes.

H8: la implementación de sistemas de información permite la competitividad de las empresas.

H9: El tamaño de la empresa está relacionado con el nivel de competitividad de la misma.

\section{Metodología}

El primer objetivo de la investigación consiste en determinar el nivel de competitividad empresarial o a nivel micro de las pymes manufactureras de Baja California. Para lo cual se desarrolló una investigación de tipo no experimental-cuantitativa, descriptiva y de corte transversal, con base en las ocho dimensiones de la competitividad empresarial del mapa de competitividad del BID.

Se diseñó un instrumento de medición compuesto por 64 preguntas $(6$ de planeación estratégica, 13 de producción y operaciones, 6 de aseguramiento de la calidad, 12 de comercialización, 7 de contabilidad y finanzas, 8 de recursos humanos, 5 de gestión ambiental y 7 de sistemas de información), el cual fue estructurado en escala de Likert, mediante cinco opciones que van desde "nunca/no se hace/ no se tiene" hasta "siempre/se aplica/se tiene". Una vez codificadas todas las respuestas por pregunta, se establecieron las puntuaciones medias para cada pregunta, posteriormente se realizó el mismo procedimiento para obtener un resultado por dimensión y luego de manera general; estos resultados se ubicaron dentro de alguno de los cinco niveles de competitividad: $1=$ muy bajo, $2=$ bajo, $3=$ mediano, $4=$ alto, $5=$ muy alto. Con estos niveles fue posible describir los resultados vertidos por el instrumento de acuerdo a lo señalado por Flores y González (2009); Moyano, Puig y Bruque (2008).

En la segunda etapa de la investigación, se desarrolla la técnica estadística de análisis de regresión lineal múltiple de tipo tradicional, la cual tiene el objetivo de encontrar qué dimensiones de las analizadas tienden a ejercer mayor influencia sobre la competitividad de las empresas. Para ello, se seleccionó como variable dependiente a la competitividad de la empresa, esta se compone de las preguntas "situación competitiva sobre el resto del sector", "frecuencia en el uso de estrategias competitivas" e "incorporación de innovación y mejoras en toda la empresa". Con el propósito de construir esta variable se hizo uso del mismo procedimiento descrito anteriormente, así se pudo conocer el valor general de la competitividad de la empresa medido en escala del 1 al 5 - muy bajo a muy alto-. 
Por otra parte, como variables independientes, se integraron las ocho dimensiones del estudio las cuales están cuantificadas en escala de Likert como se mencionó anteriormente. Asimismo, se integró la variable tamaño de la empresa como variable de control, con el objetivo de conocer si el tamaño de las empresas incide en el nivel de competitividad de las mismas. Esta variable es de tipo dicotómica donde $0=$ pequeña y $1=$ mediana.

\section{Muestra}

Para llevar a cabo la aplicación de las encuestas en las pymes manufactureras del estado, se determinaron los siguientes parámetros en la determinación de la muestra (ver la Tabla 2).

Tabla 2: Ficha técnica muestreo

\begin{tabular}{|l|c|}
\hline Población & 1004 unidades \\
\hline Muestra & 245 unidades \\
\hline Nivel de confianza & $95 \%$ \\
\hline Error & $5 \%$ \\
\hline Proporción & $70 \%$ \\
\hline Encuestas aplicadas & 195 \\
\hline
\end{tabular}

Fuente: Elaboración propia.

Por cuestiones de acceso a la información se logró encuestar a 195 empresas equivalente a casi $80 \%$ de la muestra. La aplicación del instrumento se realizó entre noviembre de 2014 y febrero de 2015, se visitó a los dueños o gerentes de las empresas para entrevistarlos y obtener resultados. En algunos casos (27) se entrevistó al gerente de recursos humanos o de producción, aquí podrían existir ciertos sesgos en determinadas dimensiones dado el nivel de conocimiento de dichos gerentes sobre su área. Otra limitación que se presentó en ciertos momentos fue la premura de las respuestas ofrecidas por falta de tiempo del entrevistado, por lo que se analizaron respuestas atípicas y casos extremos procediendo a ser eliminadas para buscar el mayor nivel de normalidad de las respuestas.

Por otra parte se validó el instrumento al realizar la prueba de confiabilidad de Alfa de Cronbach, el resultado general fue muy satisfactorio con un nivel de .98, mientras que el análisis por dimensión proporciona el siguiente resultado igualmente satisfactorio (Tabla 3).

Tabla 3: Niveles de confiabilidad por dimensión

\begin{tabular}{|l|c|}
\hline \multicolumn{1}{|c|}{ Dimensión } & Confiabilidad \\
\hline Planeación estratégica & .863 \\
\hline Producción y operaciones & .938 \\
\hline Aseguramiento de la calidad & .905 \\
\hline Comercialización & .899 \\
\hline Contabilidad y finanzas & .844 \\
\hline Recursos humanos & .891 \\
\hline Gestión ambiental & .833 \\
\hline Sistemas de información & .841 \\
\hline
\end{tabular}

Fuente: Elaboración propia. 


\section{Análisis de resultados}

Del total de empresas encuestadas, $41.7 \%$ correspondió al municipio de Tijuana, 27\% a Mexicali, 25\% Ensenada y $6.3 \%$ al municipio de Tecate. En el caso del municipio de Playas de Rosarito, este se integró al municipio de Tijuana. Asimismo, 56\% de las empresas encuestadas se catalogaron como pequeñas. Por su parte, el subsector más representado en la muestra correspondió al de productos alimenticios, bebidas y tabaco con $25 \%$, seguido del subsector de otras industrias $(22 \%)$, industrias metálicas $(13 \%)$, maquinaria y equipo $(10 \%)$, textiles, prendas de vestir e industrias del cuero $(10 \%)$ y el resto de subsectores representaron en conjunto $20 \%$. Se reconoce la no existencia de una proporcionalidad en el número de empresas encuestadas por subsector y municipio debido a las limitaciones para aceptar que las empresas participaran en el estudio. Sin embargo, los resultados son lo bastante confiables que permiten alcanzar el objetivo planteado.

Los resultados generados del estudio de competitividad empresarial en las pymes manufactureras de Baja California indican que dichas empresas se pueden dividir en dos niveles de competitividad. Por una parte, un grupo de empresas que presentan un nivel mediano (48\%); y por la otra, otro grupo (40\%) que son consideradas de bajo nivel. Muy pocas empresas presentaron niveles altos o muy altos de competitividad, indicativo de la existencia de grandes áreas de oportunidad por mejorar este sector tan significativo para la economía del estado (ver Tabla 4). Todas las dimensiones presentan bajos niveles de competitividad; sin embargo, esta se acrecienta en la dimensión de contabilidad y finanzas coincidiendo con los resultados de Saavedra y Tapia (2011), lo que sugiere que las empresas no tienen establecidos sistemas integrales para la determinación de los costos, ni llevan a cabo una planeación financiera lo suficientemente rigurosa como para tomar decisiones a corto, mediano y largo plazo en materia de costos, rentabilidad, inversión, introducción de nuevos productos, entre otras. De hecho, dentro de esta dimensión, las preguntas relacionadas con el factor costos y utilización de información financiera fueron evaluadas con niveles bajos, lo que indica que las empresas tienen carencia importante en su aplicación.

Tabla 4: Niveles de competitividad por dimensión de las pymes del sector manufacturero

\begin{tabular}{|l|c|c|c|c|c|}
\hline \multicolumn{1}{|c|}{ Nivel } & & & & \\
& Muy bajo & Bajo & Mediano & Alto & Muy Alto \\
\hline Dimensión & & & & \\
\hline Planeación estratégica & $5 \%$ & $39 \%$ & $32 \%$ & $21 \%$ & $3 \%$ \\
\hline Sistemas de información & $6 \%$ & $42 \%$ & $42 \%$ & $6 \%$ & $4 \%$ \\
\hline Producción y operaciones & $1 \%$ & $39 \%$ & $47 \%$ & $10 \%$ & $3 \%$ \\
\hline Aseguramiento de calidad & $11 \%$ & $41 \%$ & $26 \%$ & $16 \%$ & $6 \%$ \\
\hline Contabilidad y finanzas & $19 \%$ & $45 \%$ & $29 \%$ & $3 \%$ & $4 \%$ \\
\hline Comercialización & $4 \%$ & $40 \%$ & $44 \%$ & $8 \%$ & $4 \%$ \\
\hline Recursos humanos & $6 \%$ & $45 \%$ & $42 \%$ & $3 \%$ & $4 \%$ \\
\hline Gestión ambiental & $9 \%$ & $41 \%$ & $27 \%$ & $17 \%$ & $6 \%$ \\
\hline Nivel de competitividad & $\mathbf{2 \%}$ & $\mathbf{4 0 \%}$ & $\mathbf{4 8 \%}$ & $\mathbf{7 \%}$ & $\mathbf{3 \%}$ \\
\hline
\end{tabular}

Fuente: Elaboración propia con base en resultados de encuesta aplicada. 
Otras dimensiones como aseguramiento de la calidad y recursos humanos, también presentan bajos niveles de competitividad. En el primer caso, se puede tratar de una menor importancia que las empresas le dan a este factor debido a escasez de competidores, o no se considera prioritario. En el segundo caso, se identifica la falta de personal especializado en diversas áreas, así como bajo nivel de capacitación y escolaridad, y la falta de programas adecuados de selección del capital humano. Las preguntas relacionadas con contratación y selección y clima laboral fueron las peor evaluadas por las empresas. En este sentido Estrada, García y Sánchez (2009), encontraron que estas dos dimensiones no están relacionadas con el éxito competitivo en las pymes en el estado de Hidalgo. Asimismo, se puede observar que a pesar de tener en general un nivel de competitividad bajo, la dimensión de planeación estratégica es la que presenta $21 \%$ de nivel alto. Según estos datos, un determinado grupo de empresas realizan una planeación estratégica de manera continua y dan a conocer entre sus empleados los objetivos, metas, estrategias y acciones de la empresa para seguir desarrollándose. Además, son empresas que tienen por lo menos cinco años de antigüedad, lo que muestra que efectivamente aplican la planeación estratégica con cierto grado de importancia.

En lo que respecta a la dimensión de producción y operaciones, casi la mitad de las empresas presentan nivel de competitividad mediano; este resultado sugiere que las empresas consideran que la utilización de materiales y recurso humano para crear productos, contribuyen a la generación de valor para sus clientes. Los resultados obtenidos revelan que la mayoría de estas empresas realizan pronósticos de venta sobre los que basan la producción, conocen el estado y su avance.

Los niveles de competitividad por tamaño de empresa son similares entre los niveles descritos con anterioridad, aunque en el caso de las medianas prácticamente la misma cantidad se ubican en los dos niveles; en contraste, $52 \%$ de las pequeñas son medianamente competitivas respecto a $36 \%$ que son de bajo nivel.

Por otra parte, a nivel de subsectores, el de productos alimenticios, bebidas y tabaco presenta un nivel de competitividad bajo con $50 \%$ y mediano en $44 \%$. Caso muy similar se presenta en el subsector de derivados del petróleo y carbón, industrias químicas del plástico y del hule, así como al de otras industrias. El de fabricación de muebles y productos relacionados presenta un nivel mediano con 55\%. Los subsectores de industrias textiles, prendas de vestir e industrias del cuero; así como el papel, impresión e industrias relacionadas tienen un nivel de competitividad mediano en un rango de $74 \%$ y $71 \%$ respectivamente. En el caso de las industrias metálicas $12 \%$ tienen niveles altos de competitividad, aunque casi la mitad de las empresas lo son medianamente, situación que es similar al subsector de maquinaria y equipo. Un caso poco representativo - debido al escaso número de empresas registradas en el estado- es el de la industria de la madera, este se considera altamente competitivo. Finalmente, en el subsector de productos a base de minerales no metálicos, $60 \%$ de las empresas poseen un nivel medio de competitividad.

A continuación se presentan los resultados más significativos de las encuestas a escala municipio con el objetivo de comprender las particularidades de cada uno de ellos.

Mexicali: Se caracteriza por contar con medianas empresas de bajo nivel de competitividad $(54 \%)$, aunque se destaca un grupo menor $(30 \%)$ que presenta niveles muy altos. En el caso de las pequeñas empresas, $41 \%$ se coloca en el rango de bajo nivel de competitividad, mientras que $46 \%$ en nivel medio. En general, en todos los subsectores se presenta una oscilación entre el nivel bajo y medio, aunque con tendencia a la baja. Se destaca el subsector de maquinaria y equipo donde $66 \%$ se ubica en bajo nivel y el resto en un nivel muy alto. En seis de las ocho dimensiones, se mantiene la proporción entre empresas con bajo y mediano nivel de competitividad; sin embargo, en la dimensión de contabilidad y finanzas $50 \%$ presenta bajo nivel. Situación similar se percibe en la dimensión de gestión ambiental. 
Tijuana: Existe la misma proporción de medianas empresas que tienen un nivel de competitividad bajo y medio ( $46 \%$ cada una); mientras que $56 \%$ de las pequeñas se ubican en el nivel medio. Los subsectores de productos alimenticios, bebidas y tabaco, y otras industrias manufactureras son eminentemente poco competitivas. Por su parte, el de industria de la madera y productos a base de minerales no metálicos son ligeramente altos en competitividad; mientras que el resto de subsectores se ubica en una posición intermedia. En las dimensiones recursos humanos, comercialización, producción y operaciones, y sistemas de información el nivel de competitividad es mediano. La dimensión contabilidad y finanzas presenta el mismo comportamiento que en el municipio de Mexicali.

Ensenada: Tanto empresas pequeñas como medianas presentan por igual niveles de competitividad medio y bajo. Solo dos subsectores —industrias textiles, prendas de vestir e industrias del cuero y, el de papel, impresión e industria relacionadas- tienen un nivel mediano. En el resto de subsectores existe un bajo nivel. Las dimensiones gestión ambiental, contabilidad y finanzas, recursos humanos y aseguramiento de la calidad presentan bajos niveles, el resto se mantiene con una tendencia media-baja.

Tecate: Mayoritariamente las pymes tienen niveles medios de competitividad, a diferencia del resto de municipios donde existe segmentación entre los niveles medio y bajo, aquí se observa una uniformidad en los niveles de la misma. El análisis por subsectores indica que el nivel de competitividad también se considera mediano. De igual manera que en los datos anteriores, se observa un nivel medio en seis de ocho dimensiones; donde además la de planeación estratégica se considera la más competitiva, y la de contabilidad y finanzas la menos competitiva.

Los datos muestran claramente cómo a pesar de que varias dimensiones en los resultados generales son bajos en su nivel de competitividad, la de contabilidad y finanzas es la que contiene elementos de mayor preocupación, ya que fue la de menor nivel en todos los municipios del estado sin distinción del tamaño de las empresas.

En esta segunda parte de los resultados, se analizaron los aspectos más interesantes de las diferentes dimensiones y sus indicadores que formaron parte de la investigación. En una primera aproximación, se muestran los resultados del coeficiente de correlación de Pearson aplicado a las ocho dimensiones (variables independientes) en relación con la competitividad de la empresa (variable dependiente); asimismo, se introdujo la variable de control (tamaño de la empresa) en el análisis.

Tabla 5: Coeficientes de correlación de Pearson

\begin{tabular}{|l|c|}
\hline \multicolumn{1}{|c|}{ Dimensión } & $\begin{array}{c}\text { Correlación } \\
\text { Pearson }\end{array}$ \\
\hline Competitividad & 1 \\
\hline Tamaño empresa & .036 \\
\hline Planeación estratégica & $.645^{*}$ \\
\hline Producción y operaciones & $.726^{*}$ \\
\hline Aseguramiento de la calidad & $.645^{*}$ \\
\hline Comercialización & $.624^{*}$ \\
\hline Contabilidad y finanzas & $.617^{*}$ \\
\hline Recursos humanos & $.617^{*}$ \\
\hline Gestión ambiental & $.641^{*}$ \\
\hline Sistemas de información & $.594^{*}$ \\
\hline
\end{tabular}

Nota: ${ }^{*}$ Correlación significativa al nivel 0.01 (bilateral).

Fuente: Elaboración propia con base en resultados de encuesta aplicada. 
Los resultados (Tabla 5) muestran cómo las dimensiones producción y operaciones, planeación estratégica y aseguramiento de la calidad, son las que presentan un mayor nivel de correlación con respecto a la variable capacidad competitiva.

Ahora bien, para contrastar las hipótesis formuladas, se construyó un modelo de regresión múltiple, el cual busca generar mayor información que la brindada por el análisis de correlaciones. Con el objetivo de buscar mayor solidez dentro del análisis, primeramente se analizó el tipo de relación existente entre las variables mediante la utilización del diagrama de dispersión y se encontró que existe una relación lineal entre las variables, supuesto esencial para la utilización del método de mínimos cuadrados ordinarios; posteriormente se aplicó la regresión mediante el método stepwise, de esta forma se pudo determinar que de las ocho variables independientes introducidas en el análisis, solo cuatro permiten explicar mejor a la variable dependiente garantizando así la existencia de no colinealidad de las variables independientes, encontrándose los estadísticos de colinealidad (como son los coeficientes de determinación, tolerancia y factores de inflación de la varianza) dentro de los parámetros aceptados (ver tablas 6 y 7 ). Asimismo, Belsley (1991) señala que un índice de condición de hasta 10 indica un nivel de colinealidad débil y superior a 30 moderado, en este caso el índice fue de 15.45, lo que corrobora la existencia de no colinealidad como se mencionó anteriormente.

Tabla 6: Estadísticos de colinealidad

\begin{tabular}{|c|c|c|c|c|c|c|c|c|}
\hline \multirow{2}{*}{\multicolumn{2}{|c|}{ Modelo }} & \multicolumn{2}{|c|}{$\begin{array}{l}\text { Coeficientes no } \\
\text { estandarizados }\end{array}$} & \multirow{2}{*}{$\begin{array}{c}\begin{array}{c}\text { Coeficientes } \\
\text { tipificados }\end{array} \\
\text { Beta }\end{array}$} & \multirow{2}{*}{$\mathrm{T}$} & \multirow{2}{*}{ Sig. } & \multicolumn{2}{|c|}{$\begin{array}{l}\text { Estadísticos de } \\
\text { colinealidad }\end{array}$} \\
\hline & & $\mathrm{B}$ & Error típ. & & & & Tolerancia & FIV \\
\hline \multirow{2}{*}{1} & (Constante) & -.267 & .372 & & -.718 & .474 & & \\
\hline & Producción y operaciones & 1.911 & .131 & .726 & 14.530 & .000 & 1.000 & 1.000 \\
\hline \multirow{3}{*}{2} & (Constante) & -.322 & .356 & & -.904 & .367 & & \\
\hline & Producción y operaciones & 1.416 & .171 & .538 & 8.305 & .000 & .544 & 1.837 \\
\hline & $\begin{array}{l}\text { Dimensión de gestión } \\
\text { ambiental }\end{array}$ & .527 & .123 & .278 & 4.300 & .000 & .544 & 1.837 \\
\hline \multirow{4}{*}{3} & (Constante) & -.337 & .348 & & -.967 & .335 & & \\
\hline & Producción y operaciones & 1.194 & .182 & .453 & 6.578 & .000 & .459 & 2.176 \\
\hline & $\begin{array}{l}\text { Dimensión de gestión } \\
\text { ambiental }\end{array}$ & .411 & .126 & .217 & 3.271 & .001 & .496 & 2.017 \\
\hline & Dimensión de contabilidad & .411 & .133 & .197 & 3.098 & .002 & .540 & 1.853 \\
\hline \multirow{5}{*}{4} & (Constante) & -.451 & .345 & & -1.308 & .193 & & \\
\hline & Producción y operaciones & .987 & .194 & .375 & 5.090 & .000 & .389 & 2.569 \\
\hline & $\begin{array}{l}\text { Dimensión de gestión } \\
\text { ambiental }\end{array}$ & .311 & .129 & .164 & 2.414 & .017 & .456 & 2.194 \\
\hline & Dimensión de contabilidad & .369 & .131 & .177 & 2.814 & .005 & .532 & 1.878 \\
\hline & Planeación estratégica & .377 & .138 & .184 & 2.731 & .007 & .464 & 2.157 \\
\hline
\end{tabular}

Fuente: Elaboración propia con base en resultados de encuesta aplicada. 
Tabla 7: Diagnóstico de colinealidad

\begin{tabular}{|c|c|c|c|c|c|c|c|c|}
\hline \multicolumn{9}{|c|}{ Diagnósticos de colinealidad ${ }^{\mathrm{a}}$} \\
\hline \multirow[b]{2}{*}{ Modelo } & \multirow[b]{2}{*}{ Dimensión } & \multirow[b]{2}{*}{ Autovalores } & \multirow{2}{*}{$\begin{array}{l}\text { Índice de } \\
\text { condición }\end{array}$} & \multicolumn{5}{|c|}{ Proporciones de la varianza } \\
\hline & & & & Constante & $\begin{array}{c}\text { Producción y } \\
\text { operaciones }\end{array}$ & $\begin{array}{l}\text { Dimensión de } \\
\text { gestión ambiental }\end{array}$ & $\begin{array}{l}\text { Dimensión de } \\
\text { contabilidad }\end{array}$ & $\begin{array}{l}\text { Planeación } \\
\text { estratégica }\end{array}$ \\
\hline \multirow{2}{*}{1} & 1 & 1.966 & 1.000 & .02 & .02 & & & \\
\hline & 2 & .034 & 7.654 & .98 & .98 & & & \\
\hline \multirow{3}{*}{2} & 1 & 2.911 & 1.000 & .01 & .00 & .01 & & \\
\hline & 2 & .065 & 6.705 & .50 & .00 & .54 & & \\
\hline & 3 & .024 & 11.041 & .49 & 1.00 & .46 & & \\
\hline \multirow{4}{*}{3} & 1 & 3.849 & 1.000 & .00 & .00 & .00 & .00 & \\
\hline & 2 & .076 & 7.107 & .48 & .01 & .06 & .35 & \\
\hline & 3 & .052 & 8.579 & .05 & .00 & .70 & .54 & \\
\hline & 4 & .022 & 13.150 & .46 & .99 & .23 & .11 & \\
\hline \multirow{5}{*}{4} & 1 & 4.808 & 1.000 & .00 & .00 & .00 & .00 & .00 \\
\hline & 2 & .076 & 7.941 & .46 & .01 & .06 & .36 & .00 \\
\hline & 3 & .056 & 9.301 & .13 & .00 & .33 & .56 & .13 \\
\hline & 4 & .040 & 11.008 & .10 & .00 & .54 & .00 & .68 \\
\hline & 5 & .020 & 15.450 & .32 & .99 & .07 & .08 & .19 \\
\hline
\end{tabular}

Fuente: Elaboración propia con base en resultados de encuesta aplicada.

Una vez hecha la regresión se procedió a graficar los residuos para contrastar la hipótesis de normalidad de los residuos y la hipótesis de homocedasticidad. De esta forma, el modelo 4 (4 de 4) es el que mejor explica la relación entre variables, con un $\mathrm{R}$ cuadrado ajustado de .597, y un error de tipificación de la estimación de 1.219 y la prueba Durbin-Watson de 1.619 demuestra que no existe autocorrelación o independencia de los residuos, el nivel de significación fue de .007 (ver tablas 8 y 9).

Tabla 8: Estadísticas del modelo

\begin{tabular}{|c|c|c|c|c|c|c|c|c|c|c|}
\hline \multicolumn{11}{|c|}{ Resumen del modelo ${ }^{\mathrm{e}}$} \\
\hline \multirow[b]{2}{*}{ Modelo } & \multirow[b]{2}{*}{$\mathrm{R}$} & \multirow[b]{2}{*}{$\mathrm{R}$ cuadrado } & \multirow[b]{2}{*}{$\begin{array}{c}\text { R } \\
\text { cuadrado } \\
\text { corregida }\end{array}$} & \multirow[b]{2}{*}{$\begin{array}{l}\text { Error típ. de } \\
\text { la estimación }\end{array}$} & \multicolumn{5}{|c|}{ Estadísticos de cambio } & \multirow[b]{2}{*}{ Durbin-Watson } \\
\hline & & & & & $\begin{array}{c}\text { Cambio } \\
\text { en } R \\
\text { cuadrado }\end{array}$ & $\begin{array}{c}\text { Cambio } \\
\text { en F }\end{array}$ & gl1 & g12 & $\begin{array}{c}\text { Sig. } \\
\text { Cambio } \\
\text { en F }\end{array}$ & \\
\hline 1 & $.726^{\mathrm{a}}$ & .527 & .524 & 1.324 & .527 & 211.280 & 1 & 190 & .000 & \\
\hline 2 & $.754^{\mathrm{b}}$ & .569 & .564 & 1.267 & .042 & 18.486 & 1 & 189 & .000 & \\
\hline 3 & $.768^{c}$ & .590 & .583 & 1.239 & .021 & 9.596 & 1 & 188 & .002 & \\
\hline 4 & $.778^{\mathrm{d}}$ & .605 & .597 & 1.219 & .016 & 7.457 & 1 & 187 & .007 & 1.619 \\
\hline
\end{tabular}

a. Variables predictoras: (Constante), producción y operaciones.

b. Variables predictoras: (Constante), producción y operaciones, dimensión de gestión ambiental.

c. Variables predictoras: (Constante), producción y operaciones, dimensión de gestión ambiental, dimensión de contabilidad.

d. Variables predictoras: (Constante), producción y operaciones, dimensión de gestión ambiental, dimensión de contabilidad, planeación estratégica.

e. Variable dependiente: Capacidad competitiva.

Fuente: Elaboración propia con base en resultados de encuesta aplicada. 
Tabla 9: Estadísticas del modelo

\begin{tabular}{|c|c|c|c|c|c|c|}
\hline \multicolumn{7}{|c|}{ ANOVA $^{a}$} \\
\hline & Modelo & Suma de cuadrados & gl & Media cuadrática & $\mathrm{F}$ & Sig. \\
\hline \multirow{3}{*}{1} & Regresión & 370.491 & 1 & 370.491 & 211.280 & $.000^{\mathrm{b}}$ \\
\hline & Residual & 333.175 & 190 & 1.754 & & \\
\hline & Total & 703.667 & 191 & & & \\
\hline \multirow{3}{*}{2} & Regresión & 400.176 & 2 & 200.088 & 124.605 & $.000^{\mathrm{c}}$ \\
\hline & Residual & 303.491 & 189 & 1.606 & & \\
\hline & Total & 703.667 & 191 & & & \\
\hline \multirow{3}{*}{3} & Regresión & 414.914 & 3 & 138.305 & 90.047 & $.000^{\mathrm{d}}$ \\
\hline & Residual & 288.753 & 188 & 1.536 & & \\
\hline & Total & 703.667 & 191 & & & \\
\hline \multirow{3}{*}{4} & Regresión & 425.987 & 4 & 106.497 & 71.719 & $.000^{\mathrm{e}}$ \\
\hline & Residual & 277.679 & 187 & 1.485 & & \\
\hline & Total & 703.667 & 191 & & & \\
\hline \multicolumn{7}{|c|}{$\begin{array}{l}\text { a. Variable dependiente: Capacidad competitiva. } \\
\text { b. Variables predictoras: (Constante), producción y operaciones. } \\
\text { c. Variables predictoras: (Constante), producción y operaciones, dimensión de gestión ambiental. } \\
\text { d. Variables predictoras: (Constante), producción y operaciones, dimensión de gestión ambiental, dimensión de } \\
\text { contabilidad. } \\
\text { e. Variables predictoras: (Constante), producción y operaciones, dimensión de gestión ambiental, dimensión de } \\
\text { contabilidad, planeación estratégica. }\end{array}$} \\
\hline
\end{tabular}

Fuente: Elaboración propia con base en resultados de encuesta aplicada.

En la Tabla 10, se aprecia cómo las dimensiones que más inciden sobre la competitividad de las pymes manufactureras de Baja California son producción y operaciones — coincidiendo parcialmente con los resultados de Estrada et al. (2009) — y en menor medida planeación estratégica, contabilidad y finanzas y gestión ambiental -Flores y González (2009); Red Pymes-Cumex (2010) - Como resultado de estos análisis, se aceptan las hipótesis H2, H1, H5 y H7 aunque esta última con un nivel bajo de aceptación. Por otra parte, las hipótesis H3, H4, H6 y H8 deben ser rechazadas a pesar de existir evidencia teórica que las respaldan, con particular interés en el tema de los sistemas de información. Algunas posibles explicaciones para interpretar esta información, para el caso de la dimensión aseguramiento de la calidad, consisten en que las pymes mexicanas aún no han desarrollado una amplia cultura de la calidad en sus productos y procesos a diferencia de otros países. Para el caso de los sistemas de información, la explicación más plausible es la falta de penetración de dichos sistemas en las pymes partiendo de la base de que el acceso a las tecnologías de la información y comunicación es aún baja y la existente es de uso básico (Ibarra et al., 2014). En el caso de la dimensión de comercialización, el incipiente aprovechamiento de los sistemas de información y el uso tradicional de los canales de distribución pueden ser un factor que limite la expansión e innovación de los mismos, ya que hoy en día el uso de plataformas digitales y el comercio electrónico son mecanismos importantes para comercializar productos de manera más amplia y eficiente. Finalmente, en la dimensión recursos humanos, la rotación laboral, clima laboral y falta de capacitación pueden ser problemas que afecten el uso adecuado del recurso humano en las organizaciones. 
Tabla 10: Análisis de regresión múltiple

\begin{tabular}{|c|c|c|c|c|c|}
\hline \multicolumn{6}{|c|}{ Coeficientes $^{\mathrm{a}}$} \\
\hline \multirow[t]{2}{*}{ Modelo } & \multicolumn{2}{|c|}{$\begin{array}{l}\text { Coeficientes no } \\
\text { estandarizados }\end{array}$} & \multirow{2}{*}{$\begin{array}{c}\begin{array}{c}\text { Coeficientes } \\
\text { tipificados }\end{array} \\
\text { Beta } \\
\end{array}$} & \multirow[t]{2}{*}{$\mathbf{t}$} & \multirow{2}{*}{ Sig. } \\
\hline & B & Error típ. & & & \\
\hline (Constante) & -.451 & .345 & & -1.308 & .193 \\
\hline Dimensión planeación estratégica & .377 & .138 & 184 & 2.731 & .007 \\
\hline Dimensión producción y operaciones & .987 & .194 & .375 & 5.090 & .000 \\
\hline Dimensión contabilidad y finanzas & .369 & .131 & .177 & 2.814 & .005 \\
\hline Dimensión de gestión ambiental & .311 & .129 & .164 & 2.414 & .017 \\
\hline
\end{tabular}

Fuente: Elaboración propia.

Adicionalmente, los resultados muestran cómo la variable de control "Tamaño de la empresa" no aporta significancia al análisis estadístico, por lo que no aparece dentro de las variables seleccionadas por el modelo - esta fue descartada en el segundo modelo (2 de 4) - concluyendo así la no existencia de una relación entre el tamaño de la empresa con respecto a la competitividad de la misma, por tanto se rechaza la hipótesis 9 .

\section{Conclusiones}

Los estudios sobre competitividad sistémica son considerados relevantes porque permiten conocer cómo factores exógenos y endógenos en las organizaciones inciden sobre el desarrollo de las mismas.

El caso particular de la competitividad a nivel micro o empresarial, permite tener un conocimiento específico de las actividades que desarrolla la empresa en diversas áreas. Este tipo de competitividad puede considerarse como el más importante debido a que es la misma empresa la que tiene el control directo sobre las variables que le permiten desarrollarse y afianzarse en el mercado.

Los resultados mostrados indican que las pymes manufactureras de Baja California son medianamente competitivas pero con tendencia importante hacia la baja, independientemente de su tamaño. Todas las dimensiones analizadas presentan niveles de competitividad medio y bajo, situación similar se presenta en los subsectores analizados. Algunas de las dimensiones repercuten directamente en la competitividad de las empresas como se observó en el análisis de regresión múltiple realizado. Los resultados descriptivos muestran que las áreas de contabilidad y finanzas se consideran menos al momento de hacer funcionar una empresa y no existe la visión por parte de los empresarios de utilizarla como punta de partida para lograr que la competitividad de la empresa se incremente. Sin embargo, el análisis de regresión efectuado, muestra cómo dicha dimensión incide de manera importante en la competitividad de la empresa, lo que corrobora la idea de que al no priorizar esta área, las empresas dejan de lado una parte importante de su propio crecimiento y consolidación. Asimismo, la dimensión que más contribuye a explicar la competitividad de la empresa es producción y operaciones.

Estos resultados permiten generar información importante sobre la situación que guarda el sector manufacturero en Baja California en el tema de competitividad, además los resultados pueden servir para ser contrastados con otras investigaciones realizadas en la región, los cuales arrojan resultados similares en cuanto al nivel de competitividad. 
Por ello, resulta significativo que este tipo de investigaciones se sigan generando para comprender a escala micro las falencias del aparato productivo mexicano y con ello, desarrollar políticas públicas e industriales encaminadas al crecimiento y desarrollo de los involucrados. Además, los resultados permiten comprender cómo las empresas no consideran la aportación de cada área, lo que degenera en áreas sobre apoyadas en términos humanos, financieros y de gestión; mientras que otras son vistas como secundarias y por tanto sin los recursos necesarios.

Como se indicó anteriormente, una limitante en esta investigación es la falta de proporcionalidad en las encuestas aplicadas por municipios y a nivel de subsector, lo que hace que los resultados obtenidos sean tomados con precaución.

Asimismo, estos resultados permiten abrir otras líneas de investigación a escala micro y tomar en consideración de manera desagregada cada una de las dimensiones para encontrar aquellas acciones o decisiones implementadas de forma incorrecta o no implementadas que puedan ser la clave para mejorar la competitividad.

Finalmente, es imprescindible que las empresas hagan del tema de la competitividad un referente para ellas si desean realmente ser competitivas a escala nacional e internacional. Es importante que las empresas generen un cambio en su cultura empresarial, pensar en el gobierno corporativo en lugar de la empresa tradicional como mecanismo para profesionalizar todas las áreas que integran una organización.

\section{Referencias}

Abdel, G. y Romo, D. (2004). Sobre el concepto de competitividad. Documentos de trabajo en estudios de competitividad. México, Distrito Federal: Centro de estudios de competitividad, ITAM.

Aragón, A. y Rubio, A. (2005). Factores explicativos del éxito competitivo: el caso de las PyMEs del estado de Veracruz. Revista Contaduría y Administración, (216), 35-69.

Aragón, A., Rubio, A., Serna A. y Chablé, J. (2010). Estrategia y competitividad empresarial: Un estudio en las mipymes de Tabasco. Revista Investigación y Ciencia, (47), 4-12.

Belsley, D. (1991). Conditioning diagnostics: Collinearity and weak data in regression. Nueva York: John Wiley \& Sons.

Berumen, S. (2006). Una aproximación a los indicadores de la competitividad local y factores de la producción. Cuadernos de Administración, 19(31), 145-163.

Cabrera-Martínez, A., López-López, P. y Ramírez, C. (2011). La competitividad empresarial: un marco conceptual para su estudio. Documentos de investigación. Administración de Empresas (Núm. 4). Recuperado de http://papers.ssrn.com/sol3/papers. cfm?abstract_id=2016597

Castellanos, O., Gálvez, B., Montoya, L., Lagos, Y. y Montoya, I. (2006). Medición del potencial de investigación y desarrollo de las pymes a partir de su capacidad de gestión organizacional. Revista Escuela de Administración de Negocios, (57), 159-171. Recuperado de http:/ / www.redalyc.org/articulo.oa?id=20605710

Cuevas-Vargas, H., Aguilera, L., González, M. y Servín, J. (2015). The use of icts and its relation with the competitiveness of Mexican smes. European Scientific Journal, 11(13), 294-310.

Du, J. y Banwo, A. (2015). "Promoting sme competitiveness: Lessons from China and Nigeria”. American Advanced Research in Management, 1(1), 1-12. 
Dussel, E. (2001). Un análisis de la competitividad de las exportaciones de prendas de vestir en Centroamérica utilizando los programas y la metodología cAN y MAGic. Serie estudios y perspectivas (1). Recuperado de http://repositorio.cepal.org/bitstream/ handle/11362/4875/S01030302_es.pdf?sequence=1\&isAllowed=y

Esser, K., Hillebrand, W., Messner, D. y Meyer-Stamer, J. (1996). Competitividad sistémica: Nuevo desafío a las empresas y a la política. Revista de la CEPAL, (59), 39-52. Recuperado de http:/ / hdl.handle.net/11362/37977

Estrada, R., García, D. y Sánchez, V. (2009). Factores determinantes del éxito competitivo en la pyme: Estudio empírico en México. Recuperado de http://repository.uaeh. edu.mx/bitstream/handle/123456789/11552

Flores, B. y González, F. (2009). La competitividad de las pymes morelianas. Cuadernos del CIMBAGE, 11, 85-104.

González, J. (2013). Estudio sobre la competitividad de Pymes incubadas en Empreser de México A.C. sucursal, San Quintín (Tesis de Maestría), Universidad Autónoma de Baja California, México.

Gracia, M. (2006). La competitividad sistémica: Elemento fundamental de desarrollo regional y local. Ciencia y Mar, X(29), 39-46. Recuperado de http://www.umar.mx/ revistas/29/competitividad.pdf

Hernández, R. (2001). Elementos de competitividad sistémica de las pequeñas y medianas empresas (PYME) del Istmo Centroamericano. Serie estudios y perspectivas (5). Recuperado de http://repositorio.cepal.org/bitstream/handle/11362/4972/1/S0111978_es.pdf

Ibarra, M., González, L. y Cervantes, K. (2014). El aprovechamiento de las Tics en empresas pequeñas y medianas de Baja California, México: El caso del sector manufacturero. Revista Internacional de Economía y Gestión de las Organizaciones, 3(1), 43-57.

Instituto Nacional de Estadística y Geografía (Inegi). (2009). Censo económico. México.

Instituto Nacional de Estadística y Geografía (Inegi). (2014a). Directorio Estadístico Nacional de Unidades Económicas DENUE. México: Autor.

Instituto Nacional de Estadística y Geografía (Inegi). (2014b). Sistema de Cuentas Nacionales. Base de datos. México: Autor.

Investinbaja. (s.f.). Industrial structure. Recuperado de http://www.investinbaja.gob.mx/ en/vision/industrial-structure

Labarca, N. (2007). Consideraciones teóricas de la competitividad empresarial. Omnia, 13(2), 158-184.

Martínez, M., Palos, G., León, B. y Ramos, R. (2013). Innovation and competitiveness in smes: The local experience in San Luis Potosí, México. Journal of Marketing and Management, 4(1), 74-92.

Medina, M. y Naranjo, I. (2014). Variables relevantes en el diseño de estrategias para la competitividad en pymes. Orbis. Revista Cientifica Ciencias Humanas, 9(27), 116-129.

Messner, D. (1996). Latinoamérica hacia la economía mundial: condiciones para el desarrollo de la "competitividad sistémica". Duisburg, Alemania: Instituto de Desarrollo y Paz.

Molina, V., Armenteros, M., Medina, M., Barquero, J. y Espinoza, J. (2011). Reflexión sobre la sobrevivencia de las pyme en el estado de Coahuila, México. Revista Internacional Administración Eं Finanzas, 4(1), 47-66.

Morales, M. y Castellanos, O. (2007). Estrategias para el fortalecimiento de las Pyme de base tecnológica a partir del enfoque de competitividad sistémica. Innovar. Revista de Ciencias Administrativas y Sociales, 17(29), 115-136. 
Moyano, J., Puig, B. y Bruque, S. (2008). Los determinantes de la competitividad en las cooperativas. CIRIEc-España, Revista de Economía Pública, Social y Cooperativa, (61), 233-249.

Organización para la Cooperación y el Desarrollo Económico (oECD). (1992). Technology and the Economy: The Key Relationships. París: Autor.

Oxford Bussines Group. (2014). The Report. Mexico 2014, Baja California. Recuperado de http://www.investinbaja.gob.mx/docs/en/the_report_oxford_bc.pdf

Porter, M. (1991). La ventaja competitiva de las naciones. Buenos Aires, Argentina: Javier Vergara.

ProMéxico. (2013). Pymes, eslabón fundamental para el crecimiento en México. Recuperado de http://www.promexico.gob.mx/negocios-internacionales/pymes-eslabon-fundamental-para-el-crecimiento-en-mexico.html

Red PyMEs-Cumex. (2010). Un estudio comparativo del perfil financiero y administrativo de las pequeñas empresas en México: entidades del Estado de México, Hidalgo, Puebla, Sonora y Tamaulipas. Resultados finales. Revista del Centro de Investigación, 9(33), 5-30.

Rosagel, S. (2012). Pequeña empresa, líderes en exportación. Revista manufactura. Recuperado de http://www.manufactura.mx/industria/2012/10/15/pyme-manufacturera-lider-en-exportacion

Rosto, F. (2010). General diagnosis of Mexican SME's from the perspective of the marketing practices efficiency: The importance of strategic market planning as a source of competitive advantage creation. Ide@s CONCYTEG, 5(65), 1335-1363.

Saavedra, M. y Milla S. (2012). La competitividad en el nivel micro de la mipyme en el estado de Querétaro. Trabajo presentado en el xvir Congreso Internacional de Contaduría, Administración e Informática, Universidad Nacional Autónoma de México, México.

Saavedra, M. y Tapia, B. (2011). Mejores prácticas y factores de competitividad en las micro, pequeñas y medianas empresas mexicanas, Economía, 36(32), 11-36. Recuperado de http:/ / www.redalyc.org/articulo.oa?id=195623319002

Suñol, S. (2006). Aspectos teóricos de la competitividad. Revista Ciencia y Sociedad, xxxI(2), 179-198. Recuperado de http://www.redalyc.org/pdf/870/87031202.pdf

Vázquez, G., Guerrero, J. y Núñez, T. (2014). Gestión de conocimiento, capital intelectual y competitividad en pymes manufactureras en México. Revista Retos, 7(1), 2943. Recuperado de http://dspace.ups.edu.ec/bitstream/123456789/8137/1/ Gestion\%20de\%20conocimiento\%20capital\%20intelectual\%20y\%20competitividad\%20en\%20pymes\%20manufactureras\%20en\%20Mexico.pdf

Vázquez, Y. y Arredondo, L. (2014). Importancia de las pymes en el mundo. Recomendaciones para Cuba. Revista Cubana de Economía Internacional, (3). Recuperado de https://www.academia.edu/12205576/Importancia_de_las_PyMEs_en_el_mundo._Recomendaciones_para_Cuba

World Economic Forum. (2010). The global competitiveness report 2010-2011. Geneva, Suiza: Autor.

Zevallos, E. (2003). Micro, pequeñas y medianas empresas en América Latina. Revista de la CEPAL, (79), 53-70. Recuperado de http://repositorio.cepal.org/bitstream/handle/11362/37871/RVE79_es.pdf?sequence=1\&isAllowed=y 
Manuel Alejandro Ibarra Cisneros

Mexicali, Mexicano. Doctor en Economía Industrial y Relaciones Laborales por la Universidad de Castilla La Mancha, España. Licenciado en Negocios Internacionales por la Universidad Autónoma de Baja California. Adscrito a la Coordinación de Posgrado e Investigación de la Facultad de Ciencias Administrativas de la Universidad Autónoma de Baja California. Línea de investigación: administración y desarrollo de las organizaciones. Coordinador de los libros Estudio de las organizaciones: visión de las ciencias administrativas (2016), editorial ILCSA-UABC, y Estudios interdisciplinarios en las ciencias económicoadministrativas (2016), UABC.

Lourdes Alicia González Torres

Tijuana, Mexicana. Doctora en Ciencias Económicas, Maestría en Ciencias Económicas y Licenciada en Economía por la Universidad Autónoma de Baja California. Adscrita a la Facultad de Ciencias Administrativas de la Universidad Autónoma de Baja California. Línea de investigación: administración y desarrollo de las organizaciones. Entre sus publicaciones se contempla el artículo "El impacto de las TIC's en las PYMEs de la industria manufacturera de Baja California a nivel de subsectores", revista Región y Sociedad.

María del Rosario Demuner Flores

Mexicana. Doctora en Ciencias Económico Administrativas, Maestra en Administración y Contador Público de la Universidad Autónoma del Estado de México. Universidad Autónoma del Estado de México, Facultad de Contaduría y Administración, Coordinación de Investigación y Estudios de Posgrado. Directora de RECAI Revista de Estudios en Contaduría, Administración e Informática. Líneas de investigación: capital humano, capital estructural y capital relacional, gestión estratégica y capital intelectual. Publicaciones recientes: "Alternativas de gestión del capital estructural en las instituciones de educación superior. Una propuesta de evaluación”, Cuadernos de Administración, 32(55), 2016 y el libro TI en la dinámica del conocimiento empresarial, 2015. 